\title{
Psychotherapy dropouts: Do they have poor psychological adjustment?
}

\author{
GERALD J. STAHLER and RUSSELL EISENMAN \\ Temple University, Philadelphia, Pennsylvania
}

\begin{abstract}
There has been considerable concern by both the clinical and research communities as to whether psychotherapy dropouts represent a more dysfunctional group than those who remain in treatment. To investigate this issue, we examined a self-report symptom checklist, a psychiatric diagnosis, demographic information, and a therapist-rated level of functioning measure for each of 817 patients, of whom 202 had terminated therapy in less than three sessions. Comparisons between these groups suggest that, contrary to the prevailing clinical assumptions, dropouts may indeed be better functioning than nondropouts.
\end{abstract}

The problem of patients who prematurely terminate psychotherapy (frequently referred to as psychotherapy dropouts) has been considered by some to be one of the greatest single obstacles to the effectiveness of mental health service delivery (Pekarik, 1985). Indeed, one review of the relevant literature has found that $30 \%-60 \%$ of outpatient psychotherapy clients in all settings terminate by dropping out of treatment (Baekeland \& Lundwall, 1975). Moreover, there is evidence that the majority of outpatients in community mental health centers drop out of treatment (Pekarik, 1983). There has been concern about psychotherapy dropouts from both the research and practitioner communities. A large sample loss due to therapy dropouts can seriously threaten the validity of a research design in psychotherapy or drug research (Chassan, 1967). Similarly, practitioners are concerned that dropouts may be adversely affected by their premature termination, and that there are other clinical, fiscal, and personnel costs involved (Garfield, 1978; Pekarik, 1985).

The prevailing view among psychotherapists is that dropouts may be considered less functional than patients who stay in therapy (Garfield, 1978). In fact, there is some evidence that clients who drop out of treatment after one or two sessions have among the poorest outcomes documented in the psychotherapy literature (Gottschalk, Mayerson, \& Gottlieb, 1967; Pekarik, 1983, 1985). The present study investigates the validity of the prevailing belief that psychotherapy dropouts have poorer psychological adjustment than nondropouts.

\section{METHOD}

\section{Background}

The present study was conducted within the context of a larger research project, funded by the National Institute of Mental Health (NIMH) that

The data presented here were collected under the auspices of the $\mathrm{Na}$ tional Institute of Mental Health Contract 278-78-0064(OP). The authors wish to acknowledge Herbert Rappaport, William Tash, and Richard Woy for their contributions to the initial project. Requests for reprints should be addresed to Gerald Stahler, Office of the Vice Provost for Research and Graduate Studies, Room 406, USB, Temple University, Philadelphia, PA 19122. was designed to test an outcome-based quality assurance methodology at a federally funded community mental health center. NIMH was interested in assessing the feasibility and utility of a modified version of the quality assurance system developed at the Johns Hopkins University and a pilot tested at the Columbia Medical Plan in Columbia, Maryland (Hankin, 1978; Johns Hopkins University, 1978). The present study was designed and conducted within the framework provided by the major research project.

\section{Setting}

The present study was conducted in a full-service federally funded community mental health center with a catchment area population of 185,000 . It provides mostly outpatient services to approximately 2,000 clients each year. The Center is staffed by approximately 120 employees, 75\% of whom are clinical staff, including psychologists, psychiatrists, social workers, and mental health worker therapists.

\section{Subjects}

As a part of the larger study of quality assurance conducted at the community mental health center (Stahler, 1982), three subject groups were studied: an experimental group comprising 256 patients in the demonstration quality assurance project; a concurrent control group consisting of 283 patients not involved in the quality assurance project, but who were in therapy at the same mental health center during the study period; and a prestudy control group of 276 patients who had completed treatment prior to the start of this study.

\section{Instruments}

The level of functioning instrument (LOF) (Edwards, McGuirk, \& Wilson, 1978; Ellis, 1977), a 9-item rating scale concerning the patient's current level of functioning, was developed by the Colorado Division of Mental Health Statistical Analysis and Research Section and the Colorado Treatment Outcome Task Force for use in state mental health agencies and state hospitals. The instrument is routinely completed by the therapist after the clinical intake session and at termination. For the present study, therapists also completed this form for patients in the experimental group after 2 months of treatment.

The instrument is a 50-point rating scale divided into five levels of 10 points each; a rating of $0-10$ represents good functioning, and a rating of 40-50 represents severe disruption. The following nine dimensions are on the scale: sociolegal functioning, substance use, medical/ physical health, mental processes, emotional health, personal behavior, interpersonal relationships, occupation/education/home management, and meeting basic needs. All clinicians involved in the study were thoroughly versed in use of the scale since it had been a standard form in the center for over a year. Prior to making any statistical comparisons, this scale was factor analyzed using an orthogonal (varimax) rotation that yielded three dimensions: basic life functioning, psychological functioning, and antisocial behavior. 
The symptom checklist (SCL-90) (Derogatis, Lipman, \& Covi, 1973) is a 90-item self-report rating scale covering a broad range of symptomatology and taking approximately $20 \mathrm{~min}$ to complete. Each item is rated by the patient as to how much the symptom has bothered him/her over the past week. Scores on each symptom range from 0 (not at all) to 4 (extremely), with items making up a symptom profile of nine primary dimensions: somatization, obsession-compulsion, anxiety, hostility, phobic anxiety, paranoid ideation, interpersonal sensitivity, depression, and psychoticism. In addition, three distinct global indices of distress are also scored: the general severity index, which combines information on numbers of symptoms; an intensity measure; and the positive symptom total, a compilation of number of symptoms. Hoffman and Overall (1978) have shown that summing all items on the scale produces a reliable and valid global index of psychopathology.

Demographic information about the patients was obtained from questionnaires, and the official psychiatric diagnosis on each patient was obtained from the mental health center files.

\section{Procedure}

On admission to the center, patients in the experimental group completed the SCL-90. An intake worker went over example questions with the patient before the patient completed the form. Control group patients did not receive the SCL-90. They completed an admission form and proceeded to the clinical intake.

After the administrative intake was completed, patients in both experimental and control groups were assigned a primary clinician according to availability. After interviewing the patient, the therapists rated their patients on the LOF measure and completed the admission form. Both are routine procedures at this center. Dropouts were defined as patients who terminated treatment after fewer than three sessions. More information on all aspects of this study can be found in Stahler (1982).

\section{RESULTS}

First, within the experimental group, chi-square analysis revealed no differences between dropouts $(n=83)$ and nondropouts $(n=173)$ on ethnicity $\left[\chi^{2}(4)=2.77\right.$, $p=.59]$, gender $\left[\chi^{2}(1)=1.19, p=.27\right]$, and marital status $\left[\chi^{2}(4)=1.22, p=.87\right]$. The two groups did differ on diagnosis $\left[\chi^{2}(3)=12.56, p=.005\right]$, with nondropouts having a greater proportion of psychotic patients than did dropouts $\left[\chi^{2}(1)=5.81, p=.05\right]$. Three oneway analyses of variance (ANOVAs) found no significant differences between groups on age $[F(1,254)=2.73$, $p=.09]$, educational attainment $[F(1,254)=1.56$, $p=.39]$, and average annual income $[F(1,245)=.73$, $p=.39]$. The dropouts did not differ from nondropouts on the self-report symptom checklist $[F(1,215)=1.00$, $p=.31]$. On the admission LOF measure, the two groups differed on psychological functioning $[F(1,254)=16.14$, $p=.0001]$, with dropout patients being assessed by their therapists as functioning better than nondropouts. No differences between the two groups were found on basic life functioning $[F(1,255)=.83, p=.36]$ and antisocial functioning $[F(1,254)=2.22, p=.13]$.

Second, for the concurrent control group, similar analyses were performed, with the exception of the symptom checklist, which these patients did not receive. Dropouts $(n=70)$ did not differ from nondropouts $(n=213)$ on ethnicity $\left[\chi^{2}=16, p=\right.$ n.s. $]$, gender $\left[\chi^{2}(1)=.73\right.$, $p=.39]$, marital status $\left[\chi^{2}(4)=2.82, p=.58\right]$, and diagnosis $\left[\chi^{2}(3)=3.31, p=.34\right]$. Similarly, an ANOVA indicated no differences between groups on age $[F(1,281)$
$=1.13, p=.28]$, educational attainment $[F(1,263)=$ $.29, p=.58]$, and average annual income $[F(1,228)=$ $1.10, p=.29]$. The LOF measure ANOVA revealed no significant differences between dropouts and nondropouts on basic life functioning $[F(1,279)=2.08, p=.15]$, psychological functioning $[F(1,280)=.69, p=.40]$, antisocial behavior $[F(1,279)=.38, p=.53]$, and mental processes $[F(1,281)=.30, p=.59]$.

Third, for the prestudy control group, no differences were found between dropouts $(n=49)$ and nondropouts $(n=229)$ on the demographic variables of ethnicity $\left[\chi^{2}(1)=2.63, p=.85\right]$, gender $\left[\chi^{2}(1)=1.53\right.$, $p=.21]$, marital status $\left[\chi^{2}(1)=1.61, p=.80\right]$, diagnosis $\left[\chi^{2}(3)=1.29, p=.73\right]$, age $[F(1,258)=.17$, $p=.27]$, and average annual income $[F(1,245)=.65$, $p=.41]$. On the LOF scale, dropouts were rated as functioning better than nondropouts in psychological functioning at a level that just missed the .05 level of significance $[F(1,276)=3.67, p=.06]$. The other ratings were nonsignificant: basic life functioning $[F(1,276)=.21$, $p=.64]$, antisocial behavior $[F(1,276)=.10, p=.75]$, and mental processes $[F(1,276)=.93, p=.33]$.

\section{DISCUSSION}

In contrast to much of the prevailing clinical wisdom and some empirical evidence (see Pekarik, 1985), the results of the present study suggest that psychotherapy dropouts may function better to some extent than nondropouts. It is possible that many patients who seek treatment and stay for only one or two sessions should not necessarily be considered therapy dropouts. One or two sessions may be helpful to many patients who seek treatment during acute crises. In addition, they may not be seeking long-term therapy and may have better ego resources to make use of their brief exposure to treatment. On the other hand, this study neither examined client satisfaction as a function of length of stay nor investigated the reasons for premature termination. The designation of "dropout" should perhaps not be based solely on a criterion number of sessions, as commonly employed by researchers, but based more on therapist and patient perceptions of treatment termination.

The present study suggests the importance of looking at individual differences among psychotherapy patients. Those who drop out of treatment may be systematically different from those who remain, thus resulting in a biased sample. Because of the considerable implications for research and treatment, the issue of psychotherapy dropouts clearly deserves continued investigation.

\section{REFERENCES}

BAEKELAND, F., \& LUNDWALl, L. (1975). Dropping out of treatment: A critical review. Psychological Bulletin, 82, 738-783.

Chassan, J. B. (1967). Research design in clinical psychology and psychiatry. New York: Appleton-Century-Crofts.

Derogatis, L., Lipman, R., \& Covi, L. (1973). SCL-90: An outpatient psychiatric rating scale. Psychopathology Bulletin, 9, 13-28. 
Edwards, M., McGuirk, F., \& Wilson, N. (1978, August). The Fort Logan problem screen and level of functioning instrument. Paper presented at the Annual American Psychological Association Conference, Toronto, Ontario, Canada.

Elus, R. (1977). Colorado level of functioning scale. Unpublished report, Colorado Division of Mental Health, Statistical Analysis and Research Section, Denver.

GARFIELD, S. (1978). Research on client variables in psychotherapy. In S. Garfield \& A. Bergin (Eds.), Handbook of psychotherapy and behavior change (2nd ed., pp. 191-232). New York: Wiley.

Gottschalk, L., Mayerson, P., \& Gotrlieb, A. (1967). Prediction and evaluation of outcome in an emergency brief psychotherapy clinic. Journal of Nervous \& Mental Disease, 144, 77-96.

HANkIn, J. (1978). Quality assurance and the Columbia Medical Plan. Paper presented at the Second Annual ADAMHA Conference on the Provision of Alcohol, Drug Abuse, and Mental Health Services in Health Maintenance Organizations, Rockville, MD.
Hoffman, N., \& Overall, P. (1978). Factor structure of the SCL-90 in a psychiatric population. Journal of Consulting \& Clinical Psychology, 46, 1187-1191.

Johns Hopkins University, Psychiatry Studies Program (1978). Development and testing of a methodology to assess quality of mental health care. Final report on NIMH Contract No. 278-76-0090(OP), Rockville, MD.

Pekarik, G. (1983). Follow-up adjustment of outpatient dropouts. American Journal of Orthopsychiatry, 53, 501-511.

PexarIK, G. (1985). Coping with dropouts. Professional Psychology: Research \& Practice, 16, 114-123.

STAHLER, G. (1982). An assessment of therapist rating bias and the Hawthorne effect in a program evaluation. Unpublished doctoral dissertation, Temple University, Philadelphia.

(Manuscript received for publication November 17, 1986.) 